\title{
Third-Harmonic and Intermodulation Distortion in Bulk Acoustic-Wave Resonators
}

\author{
David Garcia-Pastor ${ }^{\circledR}$, Student Member, IEEE, Carlos Collado ${ }^{\circledR}$, Senior Member, IEEE, \\ Jordi Mateu $^{\circledR}$, Senior Member, IEEE, and Robert Aigner
}

\begin{abstract}
This article discusses on the measured third-order intermodulation (IMD3) products and third harmonics (H3) appearing in a set of six different solidly mounted resonators (SMR) and bulk acoustic-wave (BAW) resonators with different shapes and stack configurations. The discussion is supported by a comprehensive nonlinear distributed circuit model that considers the nonlinear effects potentially occurring in any layer of the resonator stack. The aluminum-nitride (AIN) and silicon-dioxide $\left(\mathrm{SiO}_{2}\right)$ layers are identified as the most significant contributors to the IMD3 and H3. The frequency profile of the third-order spurious signals also reveals that, in temperature-compensated resonators, where the $\mathrm{SiO}_{2}$ layers are usually thicker, the remixing effects from the second-order nonlinear terms are the major contributors to the IMD3 and H3. These second-order terms are those that explain the second-harmonic (H2) generation, whose measurements are also reported in this article. Unique values of the nonlinear material constants can explain all the measurements despite the resonators have different shapes, resonance frequencies, and stack configurations.
\end{abstract}

Index Terms-Aluminum nitride (AIN), bulk acoustic wave (BAW), electroacoustic, nonlinear, nonlinearities, silicon dioxide $\mathrm{SiO}_{2}$, solidly mounted resonators (SMRs), thirdharmonic (H3), third-order intermodulation (IMD3), third-order intermodulation (IMD3) product.

W

\section{INTRODUCTION} low-power wide-area networks, and so on) and the new incoming standards (5G-NR, IEEE 802.11ax), the mobile communication requirements are more stringent than ever. In this scenario, acoustic-wave technology has been playing a crucial role on the development of the RF filtering stages of the current portable devices [1], allowing the inclusion of more than 40 filters per device. Among acoustic technologies, bulk acoustic-wave (BAW) configuration provides many of the filters operating around $2 \mathrm{GHz}$ and above [2].

Manuscript received May 3, 2019; revised November 15, 2019; accepted November 17, 2019. This work was supported in part by the Spanish Government under Grant TEC2017-84817-C2-2-R, Grant TEC2017-88343-C4-2-R, and Grant 2017 SGR 813, in part by the Universities and Research Secretary of the Generalitat de Catalunya, and in part by the European Social Fund under Grant 2018FI_B_00448. (Corresponding author: David Garcia-Pastor.)

D. Garcia-Pastor, C. Collado, and J. Mateu are with the Signal Theory and Communications Department, Universitat Politecnica de Catalunya, 08034 Barcelona, Spain (e-mail: david.garcia@tsc.upc.edu).

R. Aigner is with Qorvo, Inc., Orlando, FL 32703 USA.

Color versions of one or more of the figures in this article are available online at http://ieeexplore.iee.org.

Digital Object Identifier 10.1109/TMTT.2019.2955135
Without detrimental to offering exceptional frequencyselective responses, acoustic filters exhibit an inherent nonlinear response due to the nature of the piezoelectric material and all other additional layers are used to create the electrodes and the acoustic reflector in the solidly mounted resonator (SMR) configuration. Such a nonlinear response might limit the application of these filters in the current and forthcoming spectrum scenarios.

In order to give response to this major concern, accurate modeling of the nonlinear response is an essential step toward the prediction and understanding of these undesired effects. Past studies proposed different nonlinear distributed models for acoustic devices [3]-[9]. Although those approaches used different circuit models, all of them made the assumption that the unique contributor to the nonlinear response was the piezoelectric layer (AlN). However, recent studies pointed out that other layers forming the resonator can also contribute to the nonlinear response. In particular, references [10] and [11] showed that the $\mathrm{SiO}_{2}$ layers of the acoustic reflector may play a significant role on the generation of second harmonics (H2), what becomes especially relevant in temperature-compensated resonators, where $\mathrm{SiO}_{2}$ layers are thicker than that in the nontemperature-compensated resonators. Collado et al. [11] also reports on $\mathrm{H} 3$ and IMD3 measurements for a single resonator, and clearly concludes that several nonlinear sources might exist to explain their behavior. Full understanding of the origin of the nonlinear effects indeed requires the identification of all the sources contributing to the overall nonlinear manifestations.

To this aim, this article focuses on the third-order nonlinear manifestations, by performing a detailed characterization of H3 and IMD3 occurring in six different resonators. All the resonators evaluated in this article have the same stack configuration, but with different layer thicknesses and shapes. The characterization process allows to identify the direct contribution and the remixing effects into the overall IMD3 and $\mathrm{H} 3$, and it provides a unique set of second- and third-order nonlinear constants of the AlN and $\mathrm{SiO}_{2}$ that can explain all the measurements. Note that the fact of applying a unique model to emulate the behavior of several nonlinear manifestations and for different resonators supports the consistency and uniqueness of the solution.

The core of the article is organized as follows. Section II recalls the nonlinear constitutive equations and their implementation into the nonlinear distributed circuit model used in this article. Section III details on the characterized resonators 
and outlines on their broadband linear frequency response as a previous step for the nonlinear characterization process. Section IV describes the measurements of the $\mathrm{H} 2$ and third-order spurious signals, H3 and IMD3, and it discusses about the potential contributing materials to the generation of these spurious signals.

\section{Nonlinear Models}

As mentioned above, we make use of a distributed circuit model to emulate the nonlinear response of the measured SMR-BAW resonators. This model is based on the nonlinear Mason equivalent circuit of the piezoelectric layer [5] and includes the nonlinear equivalent circuits of the other layers [10], [11]. It basically consists on discretizing into elemental cells each potential contributing layer to the generation of harmonics or IMD products. Then, all the elemental cells are cascaded together to model the whole resonator. The distributed model allows capturing the field distribution along each layer of interest and the inclusion of the nonlinear sources distributed along the stack of materials. This model was extensively reported in [9] and partially recalled below for the sake of a self-contained article.

Proper modeling of each elemental cell requires the formulation of the nonlinear constitutive equations at each material. For the piezoelectric case, those equations model the relation between the different field magnitude stress, strain, electric field, and electric displacement as $T, S, E$, and $D$, respectively [5]. Those field magnitudes are related to each other by the use of different constants, being these $c^{E}, e$, and $\varepsilon^{S}$ as stiffness, piezoelectric, and dielectric constant, respectively. As detailed in [9], $S$ and $D$ field magnitudes are implemented in the nonlinear model as independent variables, giving the equations

$$
\begin{aligned}
& T=c^{D} S-\frac{e}{\varepsilon^{S}} D+T_{c} \\
& E=\frac{D-e S}{\varepsilon^{S}}-V_{c} .
\end{aligned}
$$

The nonlinear sources $T_{c}$ and $V_{c}$ are

$$
\begin{aligned}
T_{c} & =\Delta T+\frac{e}{\varepsilon^{S}} \Delta D \\
V_{c} & =\frac{\Delta D}{\varepsilon^{S}} \Delta z
\end{aligned}
$$

where $c^{D}=c^{E}+e^{2} / \varepsilon^{S}$ is the stiffened elasticity and $\Delta z$ is the thickness of an elemental cell. $\Delta T$ and $\Delta D$ are the terms defining the nonlinear behavior of the piezoelectric layer, truncated to a third-order polynomial, as follows:

$$
\begin{aligned}
& \Delta T=c_{2}^{E} \frac{S^{2}}{2}-\varphi_{3} \frac{E^{2}}{2}+\varphi_{5} S E+c_{3}^{E} \frac{S^{3}}{6}+X_{7} \frac{S E^{2}}{2}-X_{9} \frac{S^{2} E}{2} \\
& \Delta D=\varepsilon_{2}^{S} \frac{E^{2}}{2}-\varphi_{5} \frac{S^{2}}{2}+\varphi_{3} S E+\varepsilon_{3} \frac{E^{3}}{6}+X_{9} \frac{S^{3}}{6}-X_{7} \frac{S^{2} E}{2} .
\end{aligned}
$$

Those nonlinear terms are mathematically defined by different second-order $\left(c_{2}^{E}, \varphi_{3}, \varphi_{5}, \varepsilon_{2}^{S}\right)$ and third-order coefficients $\left(c_{3}^{E}, \varepsilon_{3}^{S}, X_{7}, X_{9}\right)[9]$.

Fig. 1 depicts the equivalent circuit model of an elemental cell corresponding to the equations above, where the nonlinear sources $T_{c}$ and $V_{c}$ are included in the conventional distributed

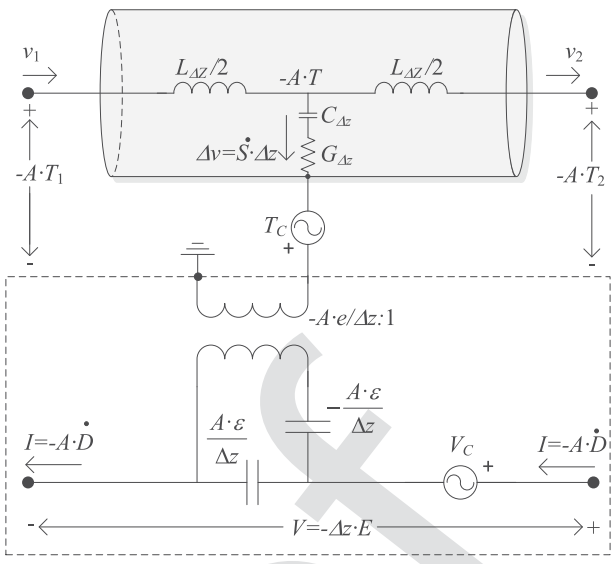

Fig. 1. Nonlinear unit cell of the piezoelectric layer [9].

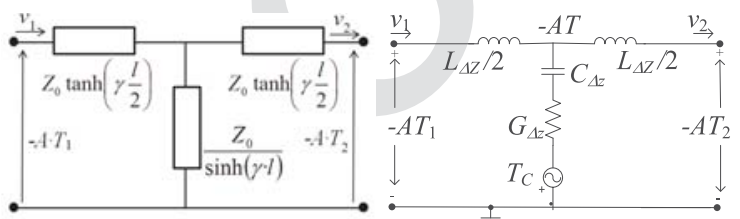

Fig. 2. Circuit models of the nonpiezoelectric layers. T-network equivalent circuit of an acoustic transmission line (left) and nonlinear unit cell (right) of a discretized transmission line [11].

Mason model [6], [9]. The number of unit cells used depends on the smallest wavelength to analyze.

For the nonpiezoelectric layers, the model to be used depends on the potential nonlinear contribution of a given material [11]. In the case of assuming a linear layer, there is no need of discretizing the layer and a T-network equivalent circuit of an acoustic transmission line can be used, as shown in Fig. 2(left). However, when the nonlinearities of the layer need to be considered, the layer is discretized as per the elemental cell shown in Fig. 2(right). In this later case, the relation of the field magnitudes $T$ and $S$, obeys

$$
T=c_{n p} S+T_{c}
$$

where $T_{c}$ is the nonlinear source and can be read as

$$
T_{c}=\frac{1}{2} c_{2, n p} S^{2}+\frac{1}{6} c_{3, n p} S^{3} .
$$

The nonlinear terms in $T_{c}$ are defined by a second-order $\left(c_{2, n p}\right)$ and a third-order $\left(c_{3, n p}\right)$ coefficient, where the subscript $n p$ indicates a given material $\left(n p=\mathrm{SiO}_{2}, W, \mathrm{AlCu}, \mathrm{SiN}\right)$.

\section{DeVICES AND LineAR RESPONSE}

This section outlines the six resonators tested in this article and their broadband measured input impedance along with the simulated impedance using the equivalent distributed model of Section II.

\section{A. Description of the Resonators}

Although being six different SMR BAW resonators, all of them present equal material distribution along the stack with different thicknesses accommodated to provide a proper linear 


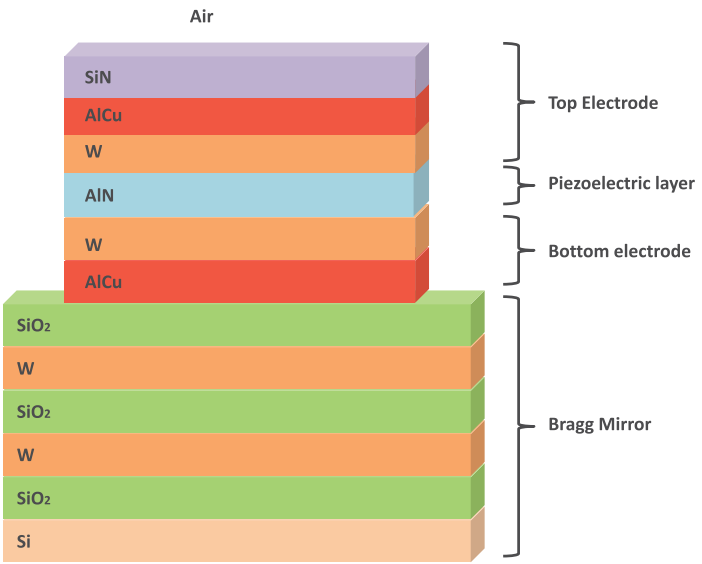

Fig. 3. Stack configuration of the measured SMR BAW devices.

TABLE I

BAW RESONATOR CHARACTERISTICS

\begin{tabular}{ccccc}
\hline Resonators & Mode & Band & Series & Shunt \\
\hline R1 & LTE-FDD & 30 & $\checkmark$ & \\
\hline R2 & LTE-FDD & 30 & & $\checkmark$ \\
\hline R3 & Wi-Fi & 2.4 GHZ & $\checkmark$ & \\
\hline R4 & Wi-Fi & 2.4 GHZ & & $\checkmark$ \\
\hline R5 & LTE-FDD & B7 & $\checkmark$ & \\
\hline R6 & LTE-FDD & B7 & & $\checkmark$ \\
\hline
\end{tabular}

response. The layer distribution of the resonator is outlined in Fig. 3. The six resonators can be classified into three groups. Each group consists of two resonators, which would correspond to a series and a shunt resonator of a ladder configuration filter. Each group of resonators has been designed to operate at different frequency ranges, which correspond to different communication services. The resonators differ on their areas, shapes, and layer thicknesses. Although the knowledge of the exact dimension of the resonators is mandatory for a proper modeling of the devices, those cannot be disclosed here for confidential reasons. Table I identifies each resonator with different names for the sake of clarity.

It is worth mentioning that R1 and R2 significantly differ from the other four resonators in the thickness of the $\mathrm{SiO}_{2}$ layers, which is set considerably thicker in order to provide a compensated temperature response.

\section{B. Linear Simulations}

An unavoidable initial step for a unified nonlinear modeling is to accurately emulate the linear broadband response of the resonator. The matching of the measured and simulated input impedances by means of a distributed model is crucial to emulate the field distributions at any point along the stack at the fundamental frequencies, $f_{1}$ and $f_{2}$, and therefore the distribution of the nonlinear sources along the stack according to (3)-(8). These nonlinear sources create spurious signals at given mixed frequencies (for example, $2 f_{1}, 3 f_{1}$, and $2 f_{1}-f_{2}$ ), whose output powers depend on how their field distributions couple to the load [12].

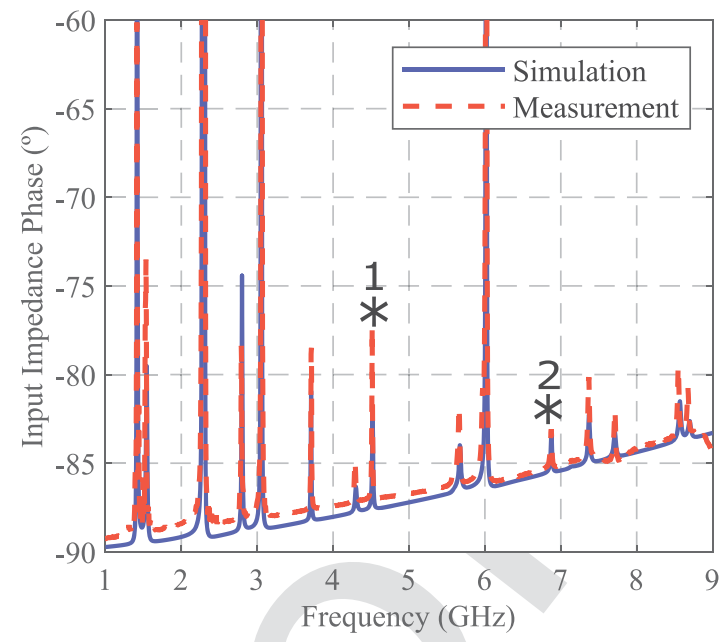

Fig. 4. Simulated and measured broadband phase frequency response of R2. Spurious resonances affecting the nonlinear response are marked with asterisks 1 and 2

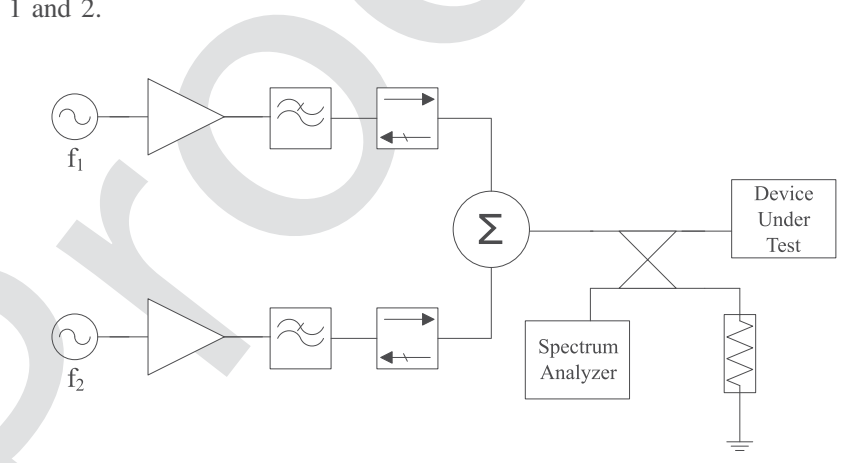

Fig. 5. H2, IMD3, and H3 measurement system.

As an example, Fig. 4 illustrates the agreement between the measured and modeled responses for resonator R2. Fine trimming within the manufacturing tolerances of layer thicknesses from the given nominal values has been performed in order to provide an accurate fitting through the whole measured frequency range. The broadband (from 1 to $9 \mathrm{GHz}$ ) input impedance phase demonstrates the accuracy of the modeling on following all the resonances appearing along the whole frequency range. Asterisks 1 and 2 in Fig. 4 indicate the resonances that have an impact on the $\mathrm{H} 2$ and $\mathrm{H} 3$ frequency responses, as it will be discussed in Section IV.

The linear response of $\mathrm{R} 1$ along with the characterization of $\mathrm{H} 2$ was reported in [11].

\section{Nonlinear Measurements}

This section provides an extensive characterization of the nonlinear response of the resonators of Table I by performing the measurements of $\mathrm{H} 2, \mathrm{H} 3$, and IMD3, using the measurement setup outlined in Fig. 5. The experiment consists of driving the resonators with two fundamental high-power tones (at $f_{1}$ and $f_{2}$ ) and measuring, using a broadband low-PIM $90^{\circ}$ hybrid coupler, the generated power reflected by the resonators. The floor level of the $\mathrm{H} 2, \mathrm{H} 3$, and IMD3 of the measurement system was obtained with the probe lifted in air, resulting in $-80,-90$, and $-90 \mathrm{dBm}$, respectively. 


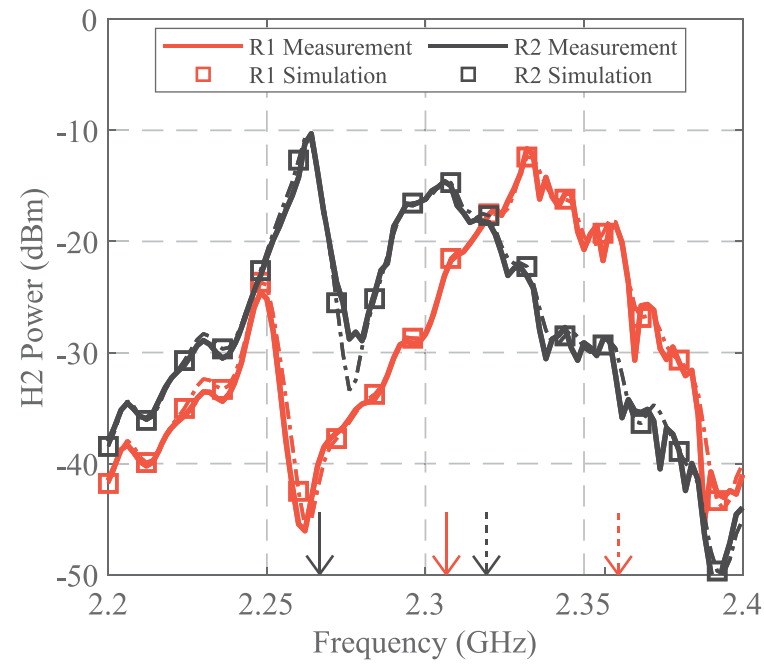

Fig. 6. $\mathrm{H} 2\left(2 \cdot f_{1}\right)$ measurements and simulations for the $\mathrm{B} 30$ resonators. Continuous and dashed arrows indicate the series and shunt resonances, respectively.

The fundamental tones are both swept over 200-MHz range and, in order to avoid potential thermal effects on the generation of IMD3, the two fundamental tones are set $10 \mathrm{MHz}$ apart in frequency [9].

From the modeling point of view, the piezoelectric layer has been discretized into 60 elemental cells and the nonpiezoelectric layers into 100 elemental cells, which guarantees to follow the field magnitude distribution even at those frequencies where a sharp variation occurs. For simplicity, adhesion layers are not included in the simulations. Their effect on the nonlinear response was shown to be negligible. The nonlinear response of the whole circuit was obtained with harmonic balance simulations using Advanced Design System.

\section{A. H2 Measurements}

Although this article focuses on the third-order nonlinear effects, measurements of the second harmonics have also been performed on the six resonators. The reason for this is twofold. First, this confirms the contribution of $\mathrm{SiO}_{2}$ layers on the generation of $\mathrm{H} 2$, which was postulated in [11]. Note that this statement was obtained from the measurements of R1, and here is confirmed with the additional measurements of R2, the other temperature-compensated resonator. Second, and as mentioned in [11], the second-order coefficients, both the $\mathrm{SiO}_{2}$ layer and the piezoelectric layer AlN, could also contribute to the generation of the third-order nonlinear effects due to a remixing process, so those coefficients need to be considered as potential contributors to the H3 and the IMD3.

Figs. 6-8 show how the second-order coefficients $\left(\varphi_{5}=-18.7 \cdot e, \varepsilon_{2}=20 \cdot \varepsilon^{S} \cdot e / c^{E}\right.$, and $\left.c_{2, \mathrm{SiO}_{2}}=-6.4 \cdot c_{\mathrm{SiO}_{2}}\right)$ published in [11] explain with good agreement the $\mathrm{H} 2$ measurements of all the resonators. The $x$-axis indicates the central frequency between the fundamental signals. As it is well known, the maximum $\mathrm{H} 2$ that appears between the series and shunt resonances (marked with arrows in the figures) is dominated by the term $\varphi_{5}$ for all the resonators, whereas the

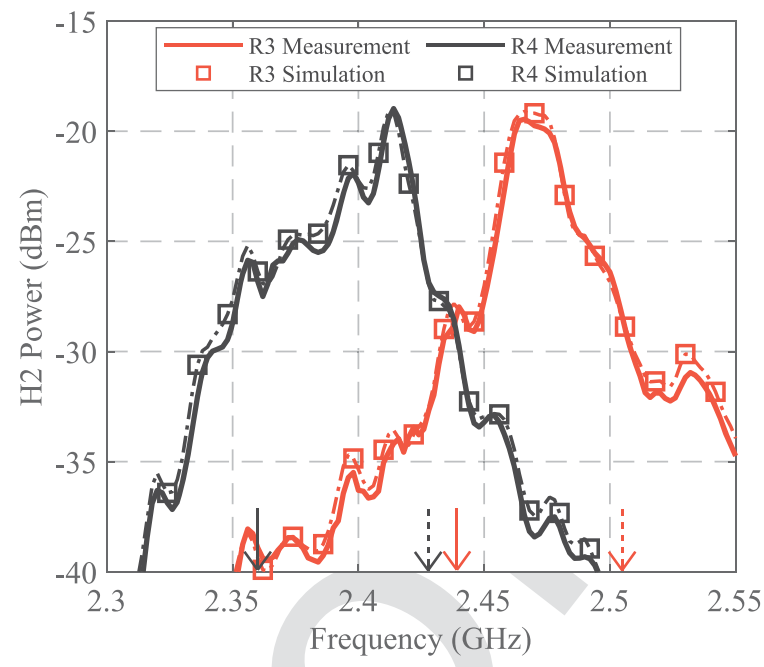

Fig. 7. $\mathrm{H} 2\left(2 \cdot f_{1}\right)$ measurements and simulations for the Wi-Fi resonators Continuous and dashed arrows indicate the series and shunt resonances, respectively.

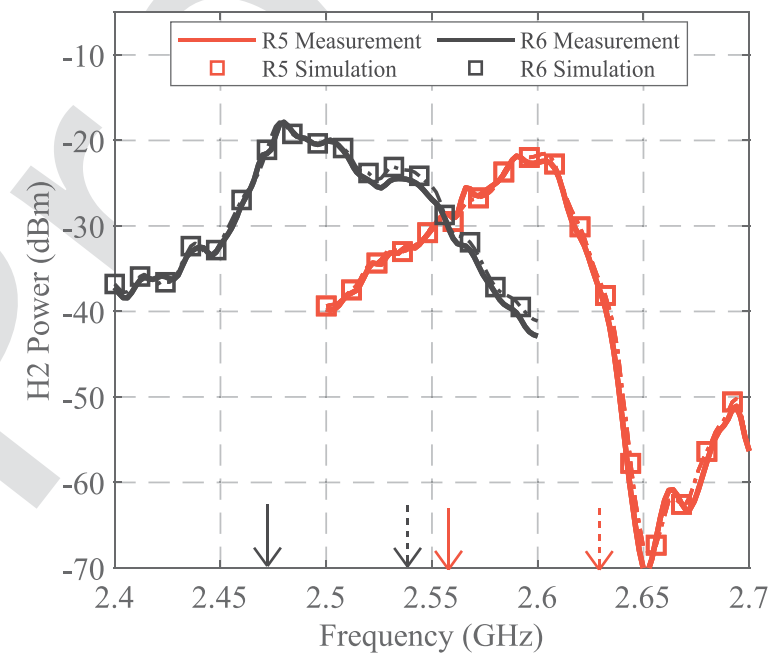

Fig. 8. H2 $\left(2 \cdot f_{1}\right)$ measurements and simulations for the B7 resonators. Continuous and dashed arrows indicate the series and shunt resonances, respectively.

term $\varepsilon_{2}$ affects to the out of band $\mathrm{H} 2$. The resonators $\mathrm{R} 1$ and $\mathrm{R} 2$ show an anomalous high $\mathrm{H} 2$ peak at 2.25 and $2.26 \mathrm{GHz}$, respectively, just below their series resonances (2.31 and $2.33 \mathrm{GHz}$ ). Those peaks are dominated by the second-order term $c_{2, \mathrm{SiO}_{2}}$ of the elastic constant of the $\mathrm{SiO}_{2}$ layers, which was set to $c_{2, \mathrm{SiO}_{2}}=-6.4 \cdot c_{\mathrm{SiO}_{2}}$ [11]. This phenomena were already reported in [11] for the R1 resonator and it appears again for the $\mathrm{R} 2$ resonator. At twice the high peak frequency $(4.50$ and $4.52 \mathrm{GHz})$, the generated $\mathrm{H} 2$ is enhanced by a high-order resonance, which can be identified with the asterisk number 1, in the input impedance of Fig. 4. Note that this also demonstrates the usefulness of using a distributed model and the importance of having a good matching between the simulations and measurements of the broadband linear response.

For R3-R6, the $\mathrm{H} 2$ response is dominated by the second-order terms coming from the AlN layer. The second-order elastic constant of the $\mathrm{SiO}_{2}$ layers only contributes to around $1 \mathrm{~dB}$ to the maximum $\mathrm{H} 2$ output power. 


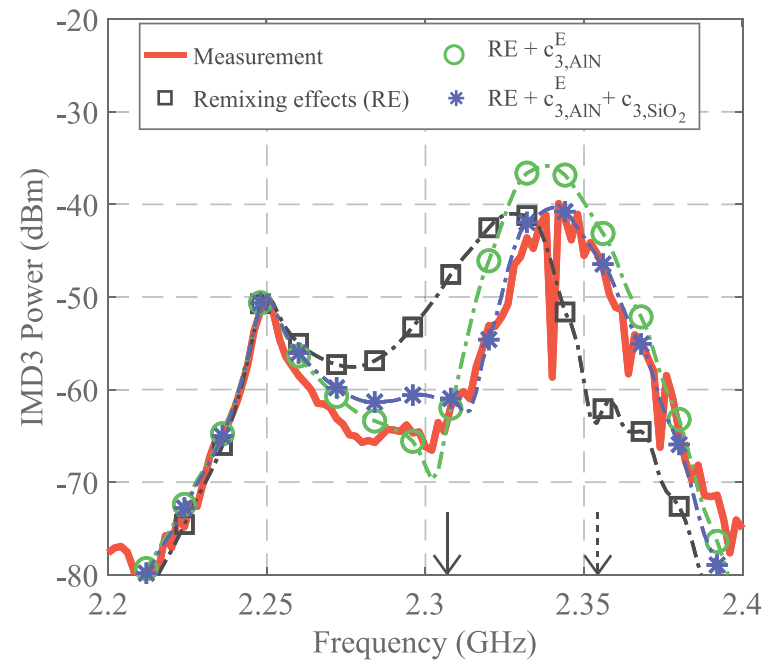

Fig. 9. IMD3 $\left(2 \cdot f_{1}-f_{2}\right)$ measurement and simulations for resonator R1. Continuous and dashed arrows indicate the series and shunt resonances, respectively.

For an accurate agreement between the measurements and the simulations, the broadband measurement system effects have been included in all the simulations. The most common effect of the nonideal measurement system is the ripple depicted in all the measurements and the reduction in the $\mathrm{H} 2$ output power at higher frequencies due to the limited bandwidth of the components used in the measurement system, which is especially relevant for the R5 resonator at frequencies higher than $2.6 \mathrm{GHz}$.

\section{B. IMD3 Measurements}

1) IMD3 Due to Remixing Effects: The next step of the characterization consists on analyzing the IMD3 of all the resonators and discerns the contribution of the second-order nonlinear terms due to the remixing phenomena.

Fig. 9 shows the measured IMD3 of the resonator R1 in thick red line. The $x$-axis corresponds to the central frequency of the two fundamental tones, i.e., $f_{0}=\left(f_{1}+f_{2}\right) / 2$, which is swept from 2.2 to $2.4 \mathrm{GHz}$. These measurements correspond to the spurious signal at $2 f_{1}-f_{2}$, when the input power level of the two fundamental tones is set to $20 \mathrm{dBm}$ and the space frequency between the two tones $\left(\Delta f=f_{2}-f_{1}\right)$ is kept to $10 \mathrm{MHz}$ along the whole experiment. Fig. 9 also shows, in squared dashed black line, the contribution to the IMD3 from the second-order nonlinear terms corresponding to AlN and $\mathrm{SiO}_{2}$ due to remixing effects. Similar measurements were reported in [11], and we concluded that the remixing effects could not solely explain the measured IMD3, because in some frequency ranges, the simulated IMD3 is higher than the measured value and in others lower. For the R2 resonator, as is depicted in Fig. 10, something similar happens and the simulated response overestimates the measurements at frequencies near the shunt resonance. Those experiments indicate that other nonlinear sources must exist beyond the remixing effects. It is remarkable that the IMD3 for these R1 and R2 exhibits an additional peak at 2.25 and $2.26 \mathrm{GHz}$, respectively, below their series resonances These peaks appear

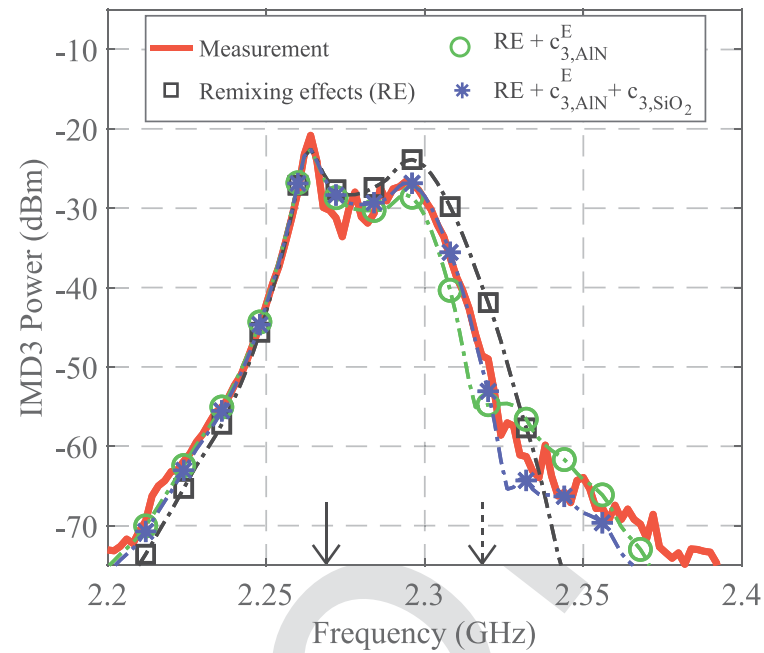

Fig. 10. IMD3 $\left(2 \cdot f_{1}-f_{2}\right)$ measurement and simulations for resonator R2. Continuous and dashed arrows indicate the series and shunt resonances, respectively.

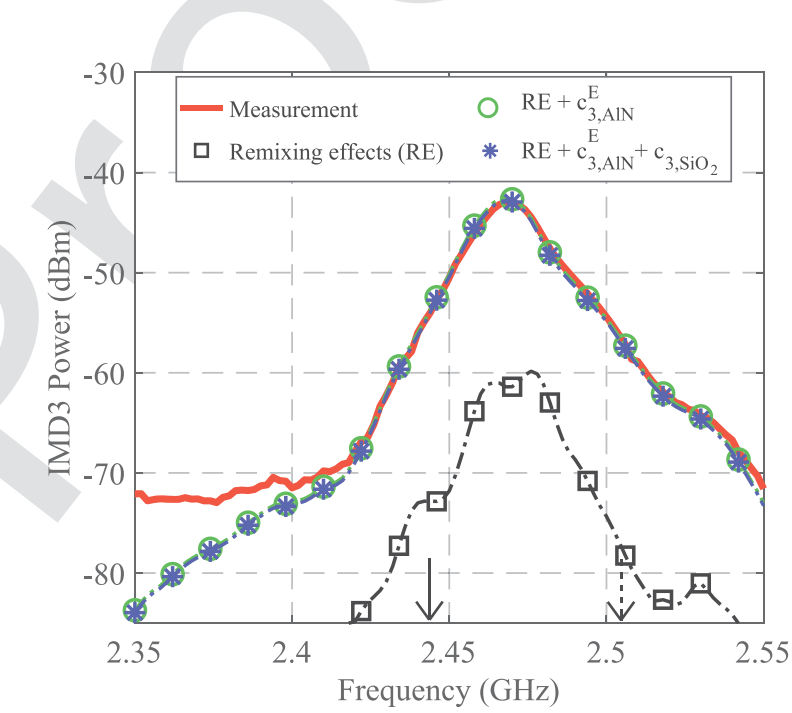

Fig. 11. IMD3 $\left(2 \cdot f_{1}-f_{2}\right)$ measurement and simulations for resonator R3. Continuous and dashed arrows indicate the series and shunt resonances, respectively.

at the same frequencies compared with those appearing in their corresponding $\mathrm{H} 2$ (see Fig. 6). It is clear then that those peaks are generated by the second-order remixing effects mainly due to the $\mathrm{SiO}_{2}$ layers.

2) IMD3 Due to AlN Third-Order Elastic Constant: To identify the third-order nonlinear terms of the different layers that additionally could contribute to the IMD3, we start by assuming that only one layer contributes to the direct generation at a time. This is setting all the third-order nonlinear constants to zero but one. We tested the potential values of $c_{3, \mathrm{AlN}}^{E}, c_{3, W}, c_{3, \mathrm{AlCu}}$, and so on, and note that for all these cases, it is always considered the contribution of the remixing effect coming from the second-order terms of $\mathrm{AlN}$ and $\mathrm{SiO}_{2}$ found in Section V. None of them adjusted all the measurements but the term $c_{3, \text { AlN }}^{E}=-110 \cdot c^{D}$ of the AlN layer. This value has been previously reported in [6] and [9] and fits perfectly the IMD3 measured of the resonators R3-R6, as it can be seen in dashed lines with green circles in Figs. 11-14. 


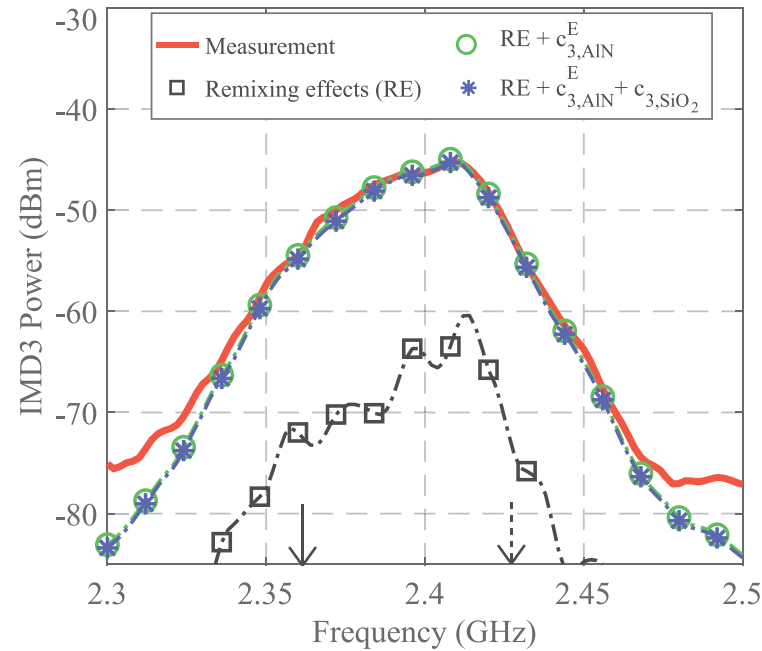

Fig. 12. IMD3 $\left(2 \cdot f_{1}-f_{2}\right)$ measurement and simulations for resonator R4. Continuous and dashed arrows indicate the series and shunt resonances, respectively.

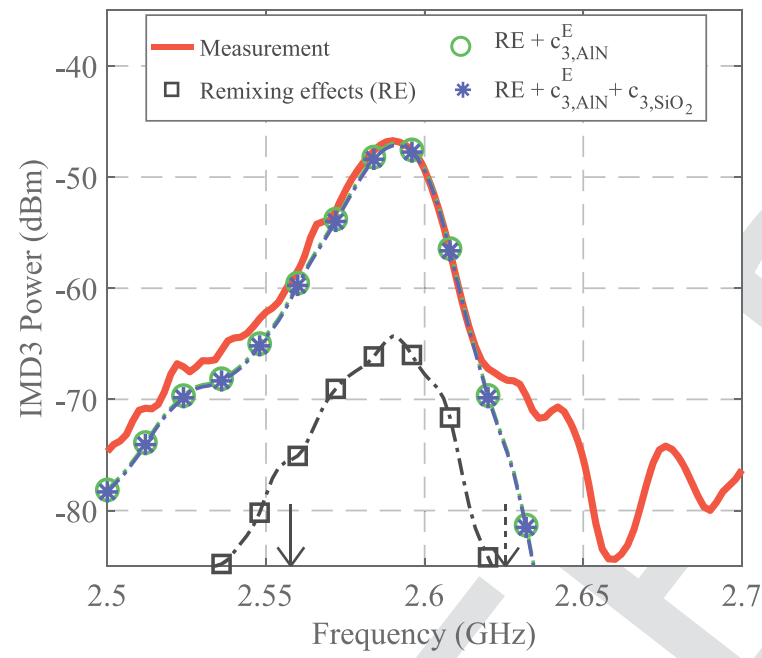

Fig. 13. IMD3 $\left(2 \cdot f_{1}-f_{2}\right)$ measurement and simulations for resonator R5. Continuous and dashed arrows indicate the series and shunt resonances, respectively.

The simulated IMD3 of the R2 resonator (see Fig. 10) also presents a better fitting with the measurements when this term is included, lowering the IMD3 that the remixing effects overestimate. For the first resonator R1, the adjustment of the IMD3 significantly improves (see Fig. 9), but still, the IMD3 is overestimated by $5 \mathrm{~dB}$ around the resonance frequency.

To capture all the nonlinear contributors fully, we look for an additional direct contribution that could affect mainly the R1 resonator and remain unchanging the IMD3 of the other resonators.

3) IMD3 Due to $\mathrm{SiO}_{2}$ Third-Order Elastic Constant: As it has been mentioned before, R1 and R2 has thicker layers of $\mathrm{SiO}_{2}$ in comparison with the other resonators. Therefore, its third-order elastic constant is the best potential candidate. Adding a value of $c_{3, \mathrm{SiO}_{2}}=30 \cdot c_{\mathrm{SiO}_{2}}$, the simulated IMD3 adjusts the experimental data as it can be seen in dashed lines with blue asterisks in Figs. 9 and 10.

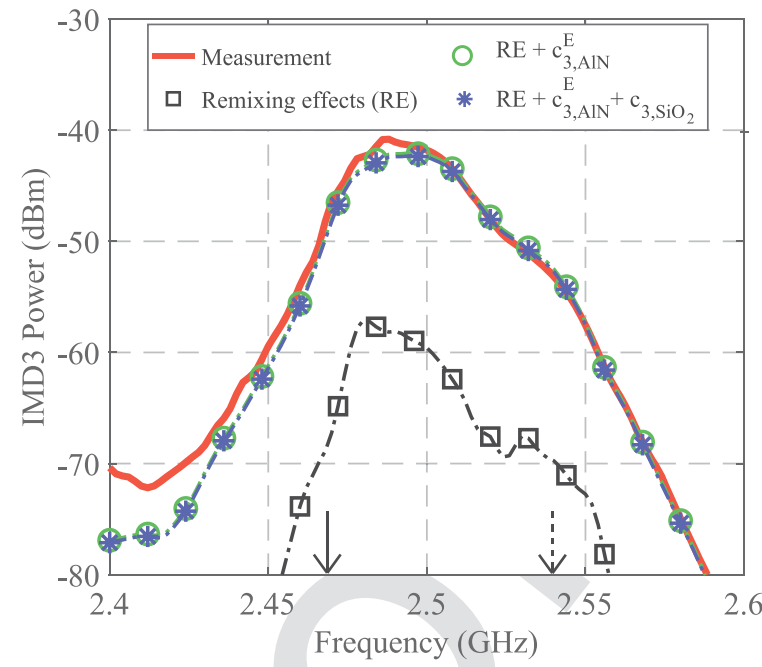

Fig. 14. IMD3 $\left(2 \cdot f_{1}-f_{2}\right)$ measurement and simulations for resonator R6. Continuous and dashed arrows indicate the series and shunt resonances, respectively.

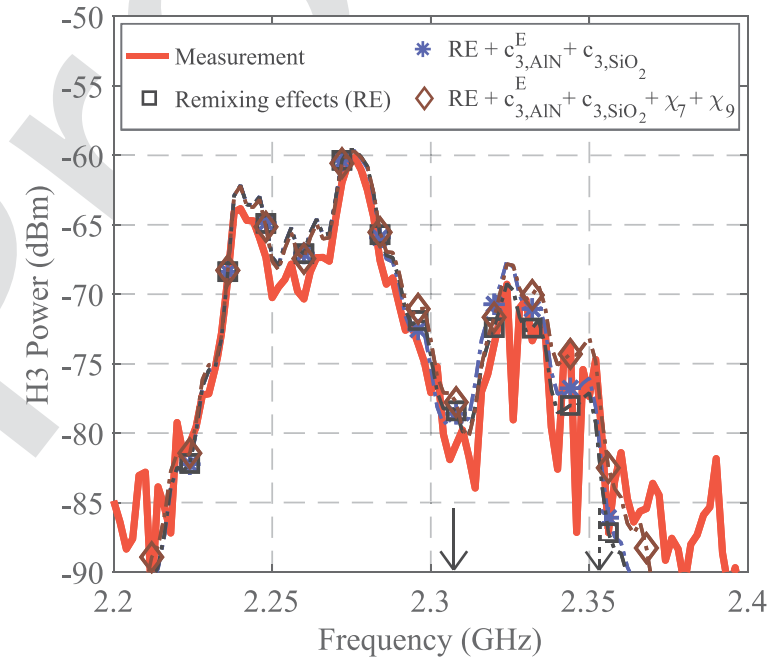

Fig. 15. H3 $\left(3 \cdot f_{1}\right)$ measurement and simulations for the test resonator R1. Continuous and dashed arrows indicate the series and shunt resonances, respectively.

Once identified this third-order nonlinear term, the other four resonators have been analyzed using the set of constants $\left(\varphi_{5}, \varepsilon_{2}^{S}, c_{2, \mathrm{SiO}_{2}}^{E}, c_{3}^{E}\right.$, and $\left.c_{3, \mathrm{SiO}_{2}}^{E}\right)$. Figs. 11-14 show that this additional term does have no impact at all into the IMD3 of those resonators.

\section{H3 Measurements}

The H3 generation must be consistent with the set of nonlinear parameters described in the previous sections.

Figs. 15 and 16 compare the measured H3 (R1 and R2) with the simulated $\mathrm{H} 3$ due to remix effects (black squares) and the set of five parameters described previously (blue asterisks), where the $x$-axis represents the fundamental frequency. It is clear that the $\mathrm{H} 3$ in the temperature-compensated resonators R1 and R2 is dominated by remixing effects. The lower frequency peaks appearing in Figs. 15 and 16 at 2.24 and 


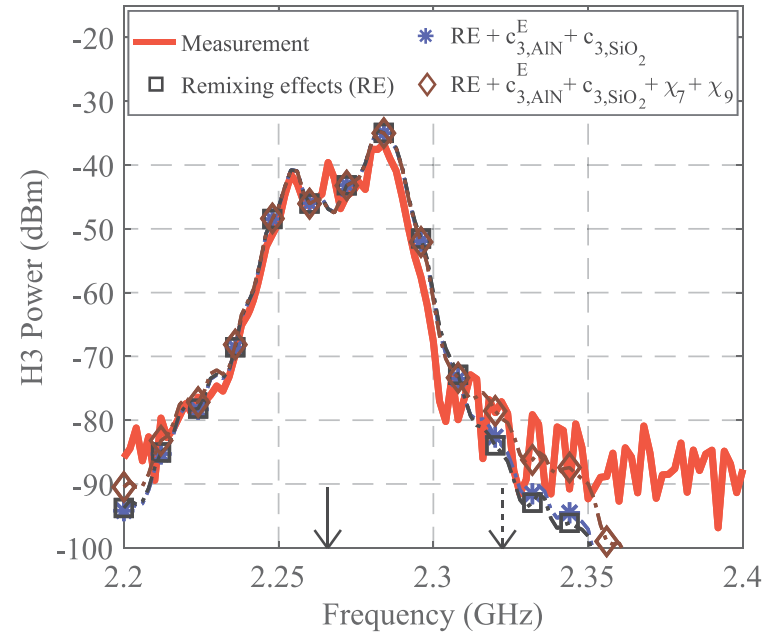

Fig. 16. $\mathrm{H} 3\left(3 \cdot f_{1}\right)$ measurement and simulations for the test resonator $\mathrm{R} 2$. Continuous and dashed arrows indicate the series and shunt resonances, respectively.

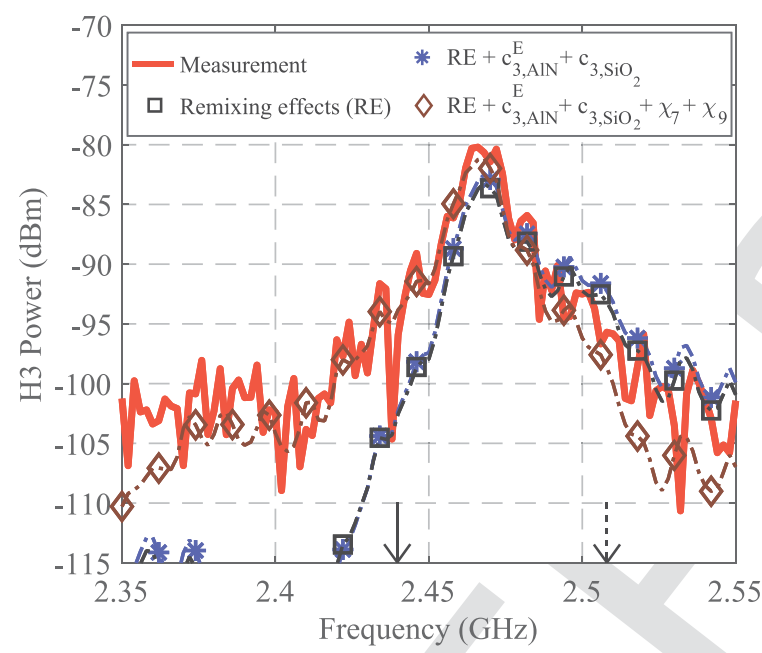

Fig. 17. $\mathrm{H} 3\left(3 \cdot f_{1}\right)$ measurement and simulations for the test resonator R3. Continuous and dashed arrows indicate the series and shunt resonances, respectively.
$2.26 \mathrm{GHz}$, respectively, have the same origin that the peaks appear at their counterparts $\mathrm{H} 2$ and IMD3. The highest peaks that appear at 2.27 and $2.28 \mathrm{GHz}$ for R1 and R2, respectively, are due to high-order resonances at 6.81 and $6.84 \mathrm{GHz}$ (depicted with the asterisk number 2 in Fig. 4) and note that the H3 does not show a conventional frequency pattern with the highest values around the resonance frequency, whereas a small hill appears around the resonance of R1 and the H3 of R2 does not show remarkable values around its resonance frequency.

The measured $\mathrm{H} 3$ of the noncompensated resonators R3-R6 (see Figs. 17-20) shows a more conventional frequency pattern with maximum values around their resonance frequencies. The simulated $\mathrm{H} 3$ of these resonators have the same order of magnitude than the measurements when the third-order terms $c_{3, \mathrm{AlN}}^{E}$ and $c_{3, \mathrm{SiO}_{2}}$ are considered (blue asterisks).

A better adjustment of the $\mathrm{H} 3$ of these four resonators can be achieved with the inclusion of additional third-order nonlinear

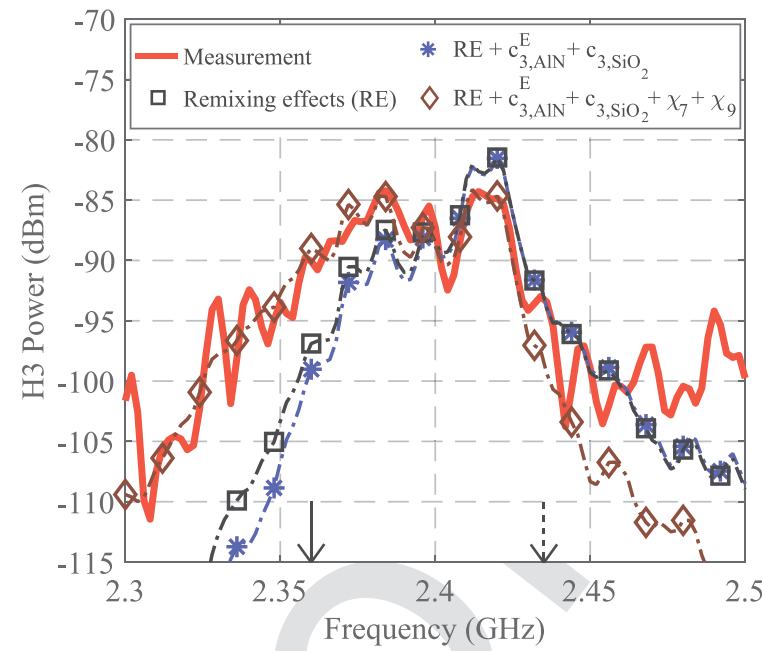

Fig. 18. H3 $\left(3 \cdot f_{1}\right)$ measurement and simulations for the test resonator R4 Continuous and dashed arrows indicate the series and shunt resonances, respectively.

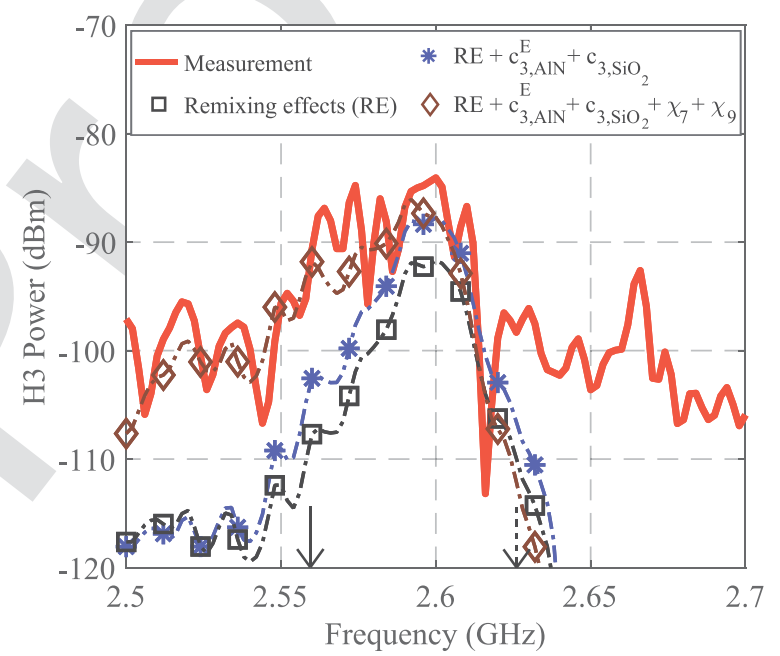

Fig. 19. H3 $\left(3 \cdot f_{1}\right)$ measurement and simulations for the test resonator R5. Continuous and dashed arrows indicate the series and shunt resonances, respectively.

constants $X_{9}=67 \cdot e$ and $X_{7}=-4 \cdot 10^{-9}$ for the AlN layer [see (5), (6)]. The term $X_{9}$ is the extension up to a third order of the term $\varphi_{5}$, which dominates the $\mathrm{H} 2$ generation around the resonance frequency, and it controls the maximum level of the H3, because $X_{9}$ multiplies $S^{3}$ in $\Delta D$ [see (6)]. The term $X_{7}$ balances the frequency pattern at both edges of the resonance frequency, since it always multiplies the electric field in $\Delta D$ and $\Delta T$ in (5) and (6).

Those two new terms $X_{9}$ and $X_{7}$ do not have an effect on the $\mathrm{H} 3$ of the $\mathrm{R} 1$ and $\mathrm{R} 2$ resonators (see brown diamonds in Figs. 15 and 16), since their $\mathrm{H} 3$ is dominated by remixing effects.

Finally, it is important to outline that the IMD3 of all the resonators is not affected by these new two third-order terms. Simulations of IMD3 considering all the terms in Table II are not included in Figs. 9-14 for the sake of clarity of the pictures, since the simulated traces would overlap the blue asterisk traces. 


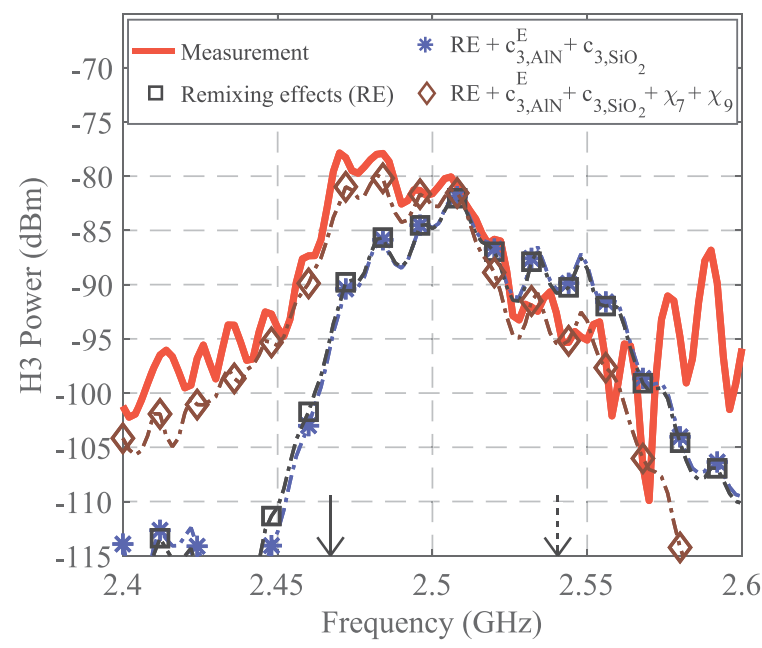

Fig. 20. H3 $\left(3 \cdot f_{1}\right)$ measurement and simulations for the test resonator R6. Continuous and dashed arrows indicate the series and shunt resonances, respectively.

TABLE II

NONLINEAR COEFFICIENTS

\begin{tabular}{cc}
\hline Nonlinear coefficient & Value \\
\hline$\varphi_{5}$ & $-18.7 \cdot e$ \\
\hline$\varepsilon_{2}$ & $20 \cdot \varepsilon^{S} \cdot e / c^{E}$ \\
\hline$c_{2, \mathrm{SiO}_{2}}$ & $-6.4 \cdot c_{S i O_{2}}$ \\
\hline$c_{3, \mathrm{AlN}}^{E} c_{3, \mathrm{SiO}}$ & $-110 \cdot c^{D}$ \\
\hline$X 9$ & $30 \cdot c_{S i O_{2}}$ \\
\hline$X 7$ & $67 \cdot e$ \\
\hline
\end{tabular}

Table II summarizes all seven nonlinear coefficients contributing to $\mathrm{H} 2, \mathrm{H} 3$, and IMD3 responses and their value.

\section{CONCLUSION}

This article outlines the major contributors into the nonlinear spurious manifestations at $\mathrm{H} 2, \mathrm{H} 3$, and IMD3, by providing a systematic characterization process and an accurate modeling of the acoustic resonators.

The modeling consisted of a distributed Mason model that has been used to successfully evaluate the second- and third-order spurious signals occurring in acoustic resonators. This provides, therefore, a unified description of the nonlinear behavior of such devices. This model has demonstrated to be valid for six different resonators evaluated in this article, which gives confidence on the uniqueness and consistency of the solution provided. The characterization process consists of a systematic procedure that allows identifying the different sources contributing to the nonlinear manifestation by sequentially adding different nonlinear contributors. This starts by the second-order nonlinear terms that explain the $\mathrm{H} 2$ values. Note that those terms also contribute to the H3 and IMD3 manifestations through a remixing phenomenon. In particular, the role of the $\mathrm{SiO}_{2}$ layers through the term $c_{2}, \mathrm{SiO}_{2}$ is crucial for the generation of IMD3 and $\mathrm{H} 3$ in the temperature-compensated resonators.

For the noncompensated resonators, our experiments confirm that the IMD3 around resonance is dominated by the term $c_{3, \mathrm{AlN}}^{E}$. However, the $\mathrm{H} 3$ is dominated by the remixing effects due to $\varphi_{5}$ and $c_{2}, \mathrm{SiO}_{2}$, and two additional third-order terms ( $X_{7}$ and $X_{9}$ ) have been included for a better adjustment of the $\mathrm{H} 3$ of all the resonators. These two additional terms do not affect to the IMD3 but additional measurements (other resonators and/or other experiments) should be performed to guarantee the uniqueness and consistence of the solution including these terms.

\section{REFERENCES}

[1] R. Ruby, "A snapshot in time: The future in filters for cell phones," IEEE Microw. Mag., vol. 16, no. 7, pp. 46-59, Aug. 2015.

2] R. Aigner, "Filter technologies for converged RF-frontend architectures: SAW, BAW and beyond," in Proc. Top. Meeting Silicon Monolithic Integr. Circuits RF Syst. (SiRF), New Orleans, LA, USA, 2010, pp. 136-139.

[3] C. Collado, E. Rocas, J. Mateu, A. Padilla, and J. M. O'Callaghan, "Nonlinear distributed model for bulk acoustic wave resonators," IEEE Trans. Microw. Theory Techn., vol. 57, no. 12, pp. 3019-3029, Dec. 2009.

[4] D. A. Feld, D. S. Shim, S. Fouladi, and F. Bayatpur, "Advances in nonlinear measurement \& modeling of bulk acoustic wave resonators (invited)," in Proc. IEEE Int. Ultrason. Symp., Chicago, IL, USA, Sep. 2014, pp. 264-272.

[5] D. S. Shim and D. A. Feld, "A general nonlinear mason model of arbitrary nonlinearities in a piezoelectric film," in Proc. IEEE Int Ultrason. Symp., San Diego, CA, USA, Oct. 2010, pp. 295-300.

[6] D. S. Shim and D. A. Feld, "A general nonlinear Mason model and its application to piezoelectric resonators," Int. J. RF Microw. Comput.Aided Eng., vol. 21, no. 5, pp. 486-495, Sep. 2011.

[7] Y. Cho and J. Wakita, "Nonlinear equivalent circuits of acoustic devices," in Proc. IEEE Int. Ultrason. Symp., Baltimore, MD, USA, vol. 2, Oct./Nov. 1993, pp. 867-872.

[8] D. A. Feld and D. S. Shim, "Determination of the nonlinear physical constants in a piezoelectric AlN film," in Proc. IEEE Int. Ultrason. Symp., San Diego, CA, USA, Oct. 2010, pp. 277-282.

[9] E. Rocas, C. Collado, J. Mateu, N. D. Orloff, J. C. Booth, and R. Aigner, "Electro-thermo-mechanical model for bulk acoustic wave resonators," IEEE Trans. Ultrason., Ferroelectr., Freq. Control, vol. 60, no. 11, pp. 2389-2403, Nov. 2013.

[10] D. Garcia-Pastor et al., "Nonlinear effects of electrode and Bragg reflector materials in BAW resonators," in Proc. IEEE Int. Ultrason. Symp., Washington, DC, USA, Sep. 2017, pp. 1-4.

[11] C. Collado et al., "Nonlinear effects of $\mathrm{SiO}_{2}$ layers in bulk acoustic wave resonators," IEEE Trans. Microw. Theory Techn., vol. 66, no. 4, pp. 1773-1779, Apr. 2018.

[12] J. Mateu, C. Collado, O. Menéndez, and J. M. O’Callaghan, "A general approach for the calculation of intermodulation distortion in cavities with superconducting endplates," Appl. Phys. Lett., vol. 82, no. 1, pp. 97-99, Jan. 2003 